\title{
Human Papillomavirus (HPV) genotype 18 variants in patients with clinical manifestations of HPV related infections in Bilbao, Spain
}

Sara L Arroyo ${ }^{1}$, Miren Basaras ${ }^{1 *}$, Elixabete Arrese ${ }^{1}$, Silvia Hernáez ${ }^{2}$, Daniel Andía ${ }^{3}$, Valentín Esteban², Koldo Garcia-Etxebarria ${ }^{4}$, Begoña M Jugo ${ }^{4}$ and Ramón Cisterna ${ }^{1,2}$

\begin{abstract}
Background: Human papillomavirus (HPV) variants differ in their biological and chemical properties, and therefore, may present differences in pathogenicity. Most authors classified variants based on the phylogenetic analysis of L1 region. Nevertheless, recombination in HPV samples is becoming a usual finding and thus, characterizing genetic variability in other regions should be essential.

Objectives: We aimed to characterize the genetic variability of HPV 18 in 5 genomic regions: E6, E7, E4, L1 and the Upstream Regulatory Region (URR), working with both single infection and multiple HPV infection samples.

Furthermore, we aimed to assess the prevalence of HPV 18 variants in our region and look for possible existence of recombination as well as analyze the relationship between these variants and the type of lesion.

Methods: From 2007 to 2010, Clinical Microbiology and Infection Control Department analyzed 44 samples which were positive for HPV 18. Genetic variability was determined in PCR products and variants were assigned to European, Asian-amerindian or African lineage. Recombination and association of variants with different types of lesion was studied.

Results: Genetic analysis of the regions revealed a total of 56 nucleotide variations. European, African and Asian-amerindian variants were found in 25/44 (56.8\%), 10/44 (22.7\%) and 5/44 (11.4\%) samples, respectively. We detected the presence of recombinant variants in 2/44 (4.5\%) cases. Samples taken from high-grade squamous intraepithelial lesions (H-SIL) only presented variants with specific-african substitutions.

Conclusions: Multiple HPV infection, non-european HPV variants prevalence and existence of recombination are considered risk factors for HPV persistence and progression of intraepithelial abnormalities, and therefore, should be taken into consideration in order to help to design and optimize diagnostics protocols as well as improve epidemiologic studies.

Our study is one of the few studies in Spain which analyses the genetic variability of HPV18 and we showed the importance of characterizing more than one genomic region in order to detect recombination and classify HPV variants properly.
\end{abstract}

Keywords: Human papillomavirus infection, Genotype 18, Variants, Recombination, Multiple infection

\footnotetext{
* Correspondence: miren.basaras@ehu.es

${ }^{1}$ Immunology, Microbiology and Parasitology Department, University of

Basque Country, Leioa 48940, Spain

Full list of author information is available at the end of the article
} 


\section{Background}

Based on the epidemiologic classification in terms of their risk to induce cervical cancer, human papillomaviruses (HPV) can be divided into 3 groups: "high-risk" genotypes associated with a greater risk of developing cancer, "low-risk" genotypes associated with low grade cell changes or benign epithelium proliferations in the genital area, but not with cancer, and "probable highrisk" genotypes from which there is not enough data about their relationship with cervical cancer to classify them [1].

About 15 genotypes are classified as high-risk types, and two of them (16 and 18) cause over $70 \%$ of all cervical cancer cases $[2,3]$. Nucleotide variability of these genotypes has been largely studied and different molecular variants were described $[4,5]$. These variants differ in their biological and chemical properties [6-8], and therefore, may become an important risk factor in cervical cancer due to possible differences in pathogenicity.

Most authors classified variants based on the phylogenetic analysis of one genomic region nucleotide variations [9]. Nevertheless, some publications have confirmed the presence of recombination in HPV samples $[10,11]$. This event may occur due to a homologous recombination or to a repeated infection of the same HPV genotype but different variant and it is more often found since coinfections with more than one HPV type are becoming a usual finding [12-14]. Therefore, it should be essential to determine HPV variants analyzing different genomic regions and multiple infections.

There are very few epidemiological national studies in Spain and all of them refer to HPV 16 which is the most investigated HPV type worldwide. However, there is no previous national work related to HPV 18 nucleotide variability, which is the second most prevalent HPV genotype found in cervical cancer.

The aim of the present study was: i) to characterize the genetic variability of HPV 18 in 5 genomic regions: E6, E7, E4, L1 and the Upstream Regulatory Region (URR), working with both single infection and multiple HPV infection samples, ii) assess the prevalence of HPV 18 variants in our region and look for existence of recombination, and iii) analyze the relationship between variants and types of lesion.

\section{Results}

\section{Samples collected}

Since 2007 to 2010, a total of 1085 positive samples for HPV were received and analyzed. HPV 18 was detected in 65 samples (6\%). Forty-four patients consented to have their samples analyzed and studied, so this study was based on their samples: 10 single HPV infections (22.7\%) and 34 multiple HPV infections (77.3\%).
We were able to amplify HPV DNA in $43 / 44$ samples for E6 region, 41/44 for E7, 35/44 for E4, 43/44 for L1 region and $44 / 44$ for URR region. All PCR products were sequenced and sequences from each region were submitted to GenBank.

\section{Nucleotide variations}

Variant distribution was determined through E6, E7, E4, L1 and URR sequences. Genetic analysis of the regions revealed a total of 56 nucleotide variations (Figure 1).

In the E6 gene nine nucleotide variations were detected. Six of them were specific to the African lineage: T317C (6/10 African variants), T251C (9/10), A548G, G266A and G374A (present in all African isolates) and C342T (5/10 African variants) which lead to a non-synonymous amino acid alteration His/Tyr. A nonsynonymous substitution $\mathrm{T} 318 \mathrm{C}$ ( $\mathrm{Tyr} / \mathrm{His}$ ) was found to be specific to the European lineage (2/25 European isolates), while the synonymous substitution C549A was detected among the three different branches (35/43 sequenced E6 amplimers). In our study, C287G was observed in all HPV 18 isolates.

E7 gene genetic variability analysis revealed five nucleotides substitutions. Three nucleotide variations were specific for the African lineage: C665T (3/10 African variants), C593T (His/Tyr), C640C and T864G (Asn/Ser). All of them but C665T and T864G were present in all African variants. One synonymous substitution (C751T) was detected in both European and Asian-amerindian isolates (26/34 non-African variants).

E4 gene analysis presented most nucleotide variations (17 substitutions) and almost half of them (8/17) lead to amino acid changes. All African variants showed 4 nonsynonymous substitutions (C3558A His/Gln, C3578T Ser/Leu, A3586C Ser/Arg and T3593G Ile/Ser), one synonymous variation (T3534C) and a deletion of 6 amino acids (3627-3632). European variants showed 4 specific non synonymous substitutions G3482A (Ser/ Asn, 3/25 isolates), T3563A (Leu/Gln, 4/25), C3617T (Ser/Leu, 4/25) and C3630G (His/Gln, all European isolates). Two non-synonymous substitutions were also detected in two European isolates: T3492A and C3615T.

$L 1$ gene and URR sequence analysis demonstrated the presence of substitutions C6842G and T7592C in all our isolates.

Most nucleotide variations found in our study have been already described in literature except for T318C, C665T, C3615T, C3617T, G6897A, G6993A, A7000T/C, T7001C, T7007G and T7765G. Only substitutions in positions 318 and 3617 lead to amino acid changes (Tir/ His and Ser/Leu, respectively). T318C substitution was present in 2 European isolates whereas C3617T nucleotide change was not specific to any lineage and was detected in 5 samples. 


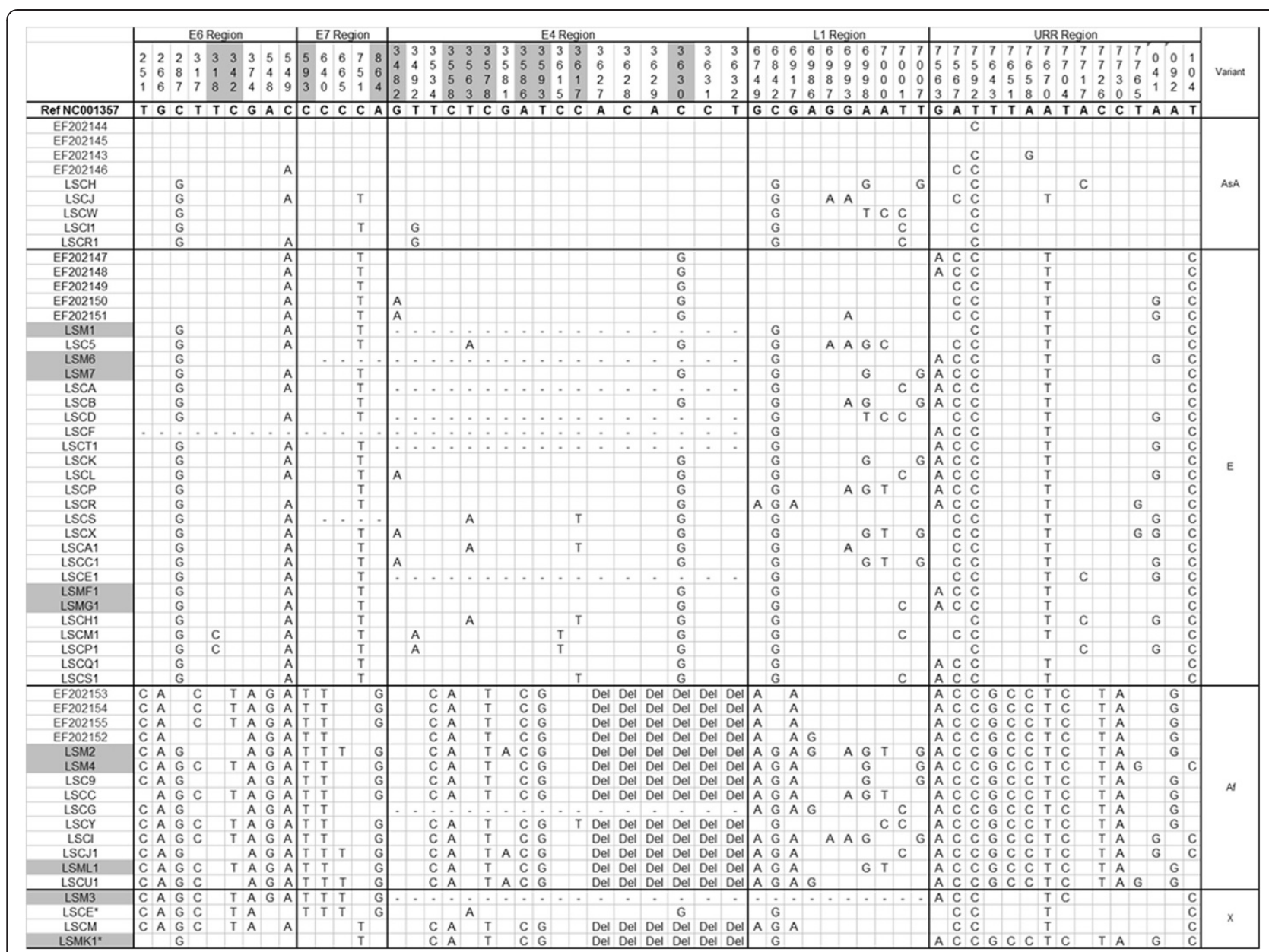

Figure 1 Nucleotide sequence variations among HPV isolates. Numbering refers to the first nucleotide of the HPV 18 reference genome (accession number NC001357). Each row indicates the isolate identification and the PCR nucleotide sequence alignment compared to the reference. Isolates EF202143-EF202155 are HPV 18 known variant sequences which belong to Asian-amerindian lineage, African and European lineage. Nucleotide positions where a substitution leads to a change of amino acid are highlighted in gray. In the first column, samples that are highlighted in gray correspond to single HPV infection samples, whereas not highlighted samples correspond to multiple HPV infections. Dashes indicate absence of nucleotide sequence data. Recombinant variants are indicated by an asterisk.

\section{HPV variants}

In our study, the predominant variant found was the European (25/44 samples) followed by the African (10/44) and the Asian-amerindian variants (5/44).

Phylogenetic analysis of the all regions studied showed that European and Asian-amerindian lineages formed closely related nodes as well as a maximal nucleotide diversity between African and non-African variants (Figure 2) (Additional file 1).

Isolates which showed nucleotide diversity from the three branches (European, Asian-amerindian and African) LSM3, LSCM, LSCE, LSMK1, LSCR, LSMH1, LSC5, LSM7 and LSCB - were analyzed for possible recombination (Table 1).

Phylogenetic trees were constructed for each sequenced region from these isolates and we found that samples LSCR, LSMH1, LSC5 and LSM7 belonged to the European branch in all regions and therefore, were classified as Europeans. Isolate LSCB belonged to the European lineage in all regions but in E6 (Asian-amerindian) due to the lack of one nucleotide substitution (C549A). This sample was also classified as European.

Samples LSM3, LSCM, LSCE, LSMK1 belonged to the African branch in some regions but were classified as European in others (Table 1).

RDP [15], Maxchi [16] and Chimaera were used for the detection of recombination in these 9 samples and only 2 of them were found to be recombinant, one single (LSMK1) and one multiple HPV infection (LCE) (Table 1). LSCM and LSM3 were classified as X variants (unknown).

\section{Variants, type of lesion and infection type}

Out of 44 samples, 32 were classified by pathologists as normal (no lesion was found), 9 samples were diagnosed 


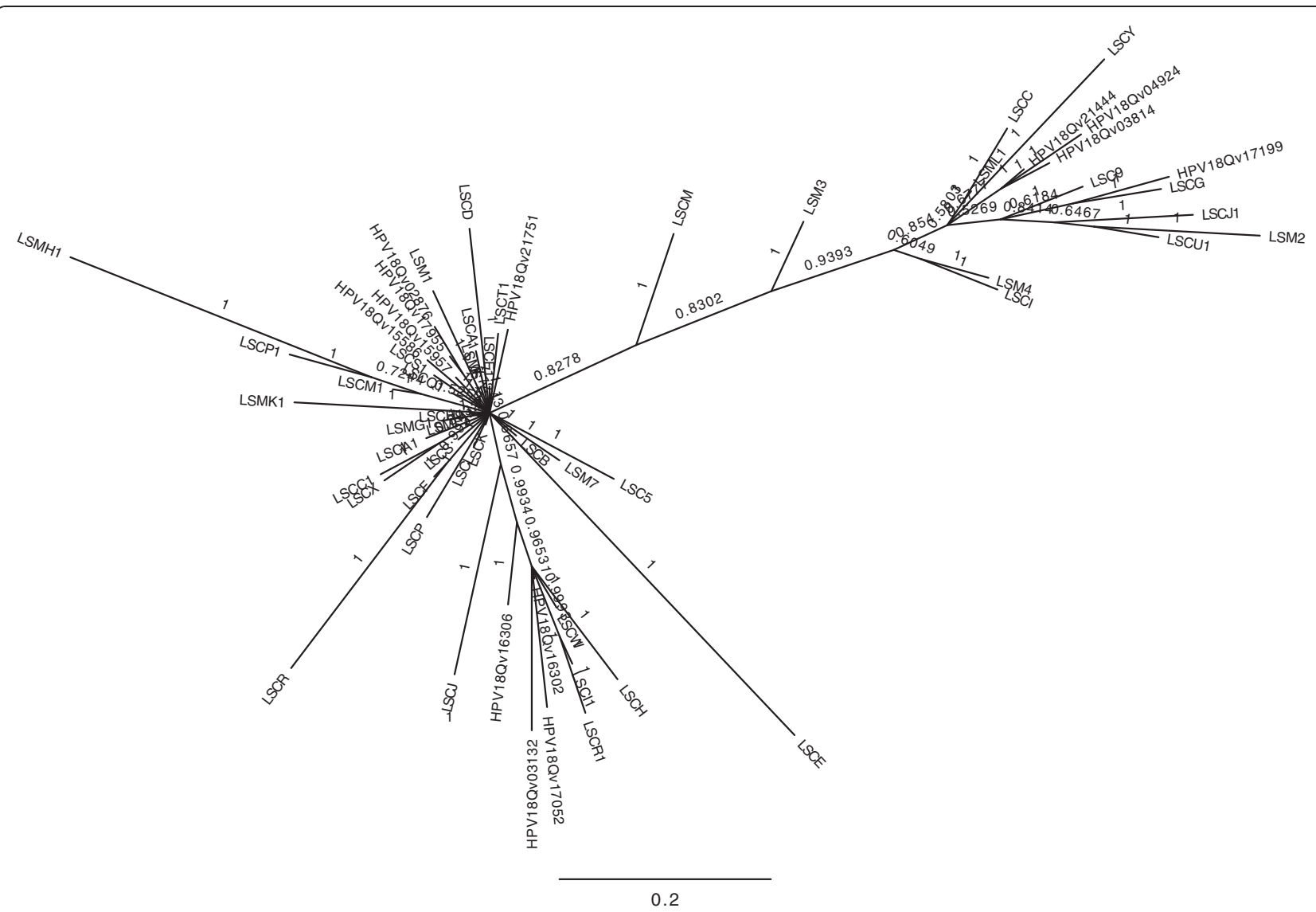

Figure 2 Phylogenetic tree of the HPV 18 isolates. E6, E7, E4, L1 and URR nucleotide sequences of isolates using the Bayesian inference method implemented in MrBayes 3.1. Isolates EF202143-EF202155 are included as HPV 18 reference variant sequences which belong to Asianamerindian lineage, African and European lineage.

as L-SIL while presence of H-SIL was detected in 3 specimens.

High grade lesions only presented African variants (2/3 isolates, 66.7\%) and variants that presented both African and European substitutions (1/3 isolates, 33.3\%) whereas most European and Asian-amerindian variants were detected in negative cytologies (Table 2).

Presence of lesions associated with non-European variants was found to be statistically significant ( $\mathrm{p}=0.01053)$. Nevertheless, there was not a statistically significant

\section{Table 1 Evidence for recombinant samples}

\begin{tabular}{|c|c|c|c|c|c|c|}
\hline Sample \Gene & E6 & E7 & E4 & L1 & URR & Program inference \\
\hline LSM3 & Af & Af & $x$ & $x$ & $E$ & No recombination detected. \\
\hline LSCE & Af & Af & E & $E$ & $E$ & Maxchi detected recombination.(LSCM is one of the donors) \\
\hline LSMK1 & AsA & $\mathrm{E}$ & Af & $E$ & Af & Maxchi detected recombination.(LSCI and LSCR1 are the donors) \\
\hline LSCR & $\mathrm{E}$ & $\mathrm{E}$ & $E$ & $E$ & $E$ & No recombination detected. \\
\hline LSMH1 & $E$ & $E$ & $E$ & $E$ & $E$ & No recombination detected. \\
\hline LSC5 & $\mathrm{E}$ & $E$ & $E$ & $E$ & $E$ & No recombination detected. \\
\hline LSM7 & $E$ & $E$ & $E$ & $E$ & $E$ & No recombination detected. \\
\hline LSCB & AsA & $E$ & $E$ & $E$ & $E$ & No recombination detected. \\
\hline LSCM & Af & $E$ & Af & Af & $E$ & No recombination detected. \\
\hline
\end{tabular}

Isolates analyzed for possible recombination. Phylogenetic trees were constructed for each sequenced region and isolates were classified as Asian-amerindian (AsA), European (E) or African (Af) variants. RDP, Maxchi and Chimaera were used for the detection of recombination. URR: Upstream regulatory region, X: absence of data. 
Table 2 Human papillomavirus 18 variants vs type of lesion

\begin{tabular}{lccc}
\hline Variants \Type of Lesion & Neg & L-SIL & H-SIL \\
\hline European & $\mathbf{7 1 . 0} \%$ & $3.3 \%$ & $0 \%$ \\
Asian-amerindian & $12.9 \%$ & $1.1 \%$ & $0 \%$ \\
African & $12.9 \%$ & $4.4 \%$ & $\mathbf{1 0 0} \%$ \\
Recombinant & $3.2 \%$ & $1.1 \%$ & $0 \%$ \\
\hline
\end{tabular}

Neg: no lesion, L-SIL: low-grade squamous intrapehitelial lesion, H-SIL: highgrade squamous intraephitelial lesion.

association between type of infections (single vs multiple) and presence of lesions $(\mathrm{p}=0.18078)$.

\section{Discussion}

There are almost no epidemiologic studies about HPV 18 variants carried out in Spain and even though national HPV prevalence is low, it cannot be forgotten that this genotype together with genotype 16 cause $70 \%$ of cervical cancer cases.

Many authors confirm that distribution of HPV variants is related to geographic or race distribution $[6,17]$ and therefore, Spain should expect predominance of European variant, followed by African and Asian-amerindian variants. Our results show concordance with this stating: 25 European variants (56.8\%), 10 African (22.7\%) and 5 Asian-amerindian variants (11.4\%).

HPV 18 has been associated with both recurrent lesions with very bad clinical prognosis [18] and benign lesions [19]. This fact may reflect the oncogenic potential difference among variants. Hecht et al [20] identified a HPV 18 variant with lower oncogenic potential due to its absence in cervical cancer but presence in $40 \%$ of intraepithelial lesions. Villa et al [21] suggested that non-European HPV 18 variants persisted more frequently and were more associated with pre-invasive lesions. Since then, most studies confirm that different variants of the same genotype differ in their pathogenic characteristics and therefore, nucleotide substitutions may play an important role. Our study results show concordance with these statements as African variants and variants where most of specificafrican substitutions were detected were the only type of variants detected in $\mathrm{H}$-SIL.

Most nucleotide changes reported in our study have been previously described and some of them are of particular importance. In URR, the mutation A41G is located in the Sp-1 binding site and isolates with this nucleotide variation have shown to have an increased transcriptional activity [22]. Variations in positions 41 and 104 modulate Sp1 and YY1 activities and are associated to a higher activity of the E6/E7 promoter. Patients with T104C substitution are less likely to present tumour recurrence [23]. Other nucleotide changes like T7651C, A7658C and C7726T also lay within transcription factor binding sites.

Substitutions C287G, C6842G and T7592C were found in all our isolates. Variation C6842G has been previously reported as error in the original sequence [24], and $\mathrm{H}$. Arias-Pulido et al. sequenced the original reference HPV 18 plasmid (provided by E-M de Villiers, Deutsches Krebsforschungzentrum, Germany) and observed the substitution T7592C [25], so it is considered as a sequencing error in the original HPV 18 reference sequence report.

Furthermore, ten "new" nucleotide variations have been detected and two of them were non-synonymous and lead to amino acid changes (T318C and C3617T, Tir/His and Ser/Leu, respectively).

Knowledge on HPV variants and their nucleotide variability is essential for three main reasons: i) nucleotide variations may interfere with the viral oncogenic potential, ii) host cellular immune response can be different when

Table 3 Polymerase chain reaction characteristics for Human papillomavirus 18

\begin{tabular}{|c|c|c|c|c|}
\hline & Primer sequence $\left(5^{\prime}-3^{\prime}\right)$ & Annealing Temp/Cycles & Nucleotides amplified* & Amplicon size \\
\hline E6 F & AGTAACCGAAAACGGTCGGGA & $55^{\circ} \mathrm{C} / 40$ cycles & $38-491$ & $454 \mathrm{pb}$ \\
\hline E6 R & GTTGTGAAATCGTCGTIITCA & & & \\
\hline E7 F & TGAAAAACGACGATTTCACAAC & $55^{\circ} \mathrm{C} / 40$ cycles & $470-931$ & $462 \mathrm{pb}$ \\
\hline E7 R & ACCTTCTGGATCAGCCATTG & & & \\
\hline E4 F & GTAAAGGAAGGGTACAACACG & $57^{\circ} \mathrm{C} / 35$ cycles & 3309-3792 & $484 \mathrm{pb}$ \\
\hline E4 R & CTGTCCAATGCCAGGTGGA & & & \\
\hline LCR $1 \mathrm{~F}$ & TCGGTTGCCTITGGCTTAT & $55^{\circ} \mathrm{C} / 40$ cycles & $7465-7775$ & $311 \mathrm{pb}$ \\
\hline LCR $1 \mathrm{R}$ & AAGGGTAGACAGAATGTTGGACA & $55^{\circ} \mathrm{C} / 40$ cycles & $7718-163$ & $303 \mathrm{pb}$ \\
\hline LCR 2 F & GCTAATTGCATACTTGGCTTG & & & \\
\hline LCR 2 R & TCCGTGCACAGATCAGGTAG & & & \\
\hline MY11 & GCACAGGGTCATAACAATGG & $55^{\circ} \mathrm{C} / 40$ cycles & 6558-7012 & $455 \mathrm{pb}$ \\
\hline MY09 & CGTCCAAGGGATATTGATC & & & \\
\hline L1 seq & ACAGTCTCCTGTACCTGGG & & & \\
\hline
\end{tabular}

*Position numbering refers to the first nucleotide of the HPV 18 reference genome (accession number NC001357). F: forward primer, R: reverse primer. 
Table 4 GeneBank accession numbers for the sequenced isolates

\begin{tabular}{|c|c|c|c|c|c|}
\hline Isolate & E6 & E7 & E4 & L1 & URR \\
\hline LSM1 & JN416211 & JN416162 & $x$ & JN416262 & JN416313 \\
\hline M2 & JN416212 & JN416163 & JN416121 & N416263 & N416314 \\
\hline SM3 & JN416213 & JN416164 & $x$ & $x$ & JN416315 \\
\hline SM4 & JN416214 & JN416165 & JN416122 & JN416264 & JN416316 \\
\hline $5 \mathrm{C} 5$ & JN416215 & JN416166 & JN416123 & JN416265 & JN416317 \\
\hline SM6 & JN416216 & $x$ & $x$ & JN416266 & JN416318 \\
\hline SM7 & & JN416167 & JN416124 & JN416 & JN41631S \\
\hline SC9 & JN416219 & JN416169 & JN416125 & JN416269 & JN416321 \\
\hline sca & JN416220 & JN416170 & $x$ & JN416270 & JN416322 \\
\hline SCB & JN416221 & JN416 & JN416126 & JN416271 & 6323 \\
\hline SCC & JN416222 & JN416172 & JN416127 & JN416272 & JN416324 \\
\hline SCD & JN416223 & JN416173 & $x$ & JN416273 & JN416325 \\
\hline SCE & JN416224 & JN416174 & JN416128 & JN416274 & JN416326 \\
\hline $\mathrm{SCF}$ & $x$ & $x$ & $x$ & JN416275 & \\
\hline SCG & JN416225 & JN416175 & $x$ & JN416276 & JN416328 \\
\hline SCH & JN416226 & JN416176 & JN416129 & JN416277 & 6329 \\
\hline $\mathrm{SCl}$ & JN416227 & JN416177 & JN416130 & JN416278 & JN416330 \\
\hline SCJ & JN416228 & JN416178 & JN416131 & JN416279 & JN416331 \\
\hline SCK & JN416 & JN41 & & & \\
\hline $\mathrm{CL}$ & JN416230 & JN416180 & JN416133 & JN416281 & \\
\hline SCM & & & & & \\
\hline SCP & JN416232 & JN41C & & & \\
\hline SCR & JN416234 & JN416184 & JN416137 & JN416285 & JN416337 \\
\hline LSCS & $\mathrm{JN} 416$ & $x$ & & & \\
\hline SCW & JN416238 & JN416187 & JN41 & JN41 & \\
\hline $\operatorname{ccy}$ & JN416239 & JN416188 & JN416142 & & JN416342 \\
\hline SCY & JN416240 & JN416189 & JN416 & JN41C & $\mathrm{JN}$ \\
\hline SCA1 & JN416241 & JN416190 & JN416144 & & \\
\hline SCC1 & JN416243 & JN416192 & JN416146 & & JN416346 \\
\hline SCE1 & JN416245 & JN416194 & $x$ & JN416296 & 6348 \\
\hline $5 M=1$ & JN416246 & JN416195 & JN416147 & & \\
\hline SMG1 & JN416247 & JN416196 & JN416148 & & JN416350 \\
\hline $\mathrm{SCH} 1$ & JN416248 & JN416197 & JN416149 & JN416299 & JN416351 \\
\hline L्रा1 & JN416249 & JN416198 & JN416150 & JN416300 & JN416352 \\
\hline LSCJ1 & JN416250 & & JN416151 & JN416301 & JN41 \\
\hline LSMK1 & JN416251 & JN416200 & JN416152 & JN416302 & JN416354 \\
\hline SIVILI & JN416252 & JN416201 & JN416153 & JN416303 & \\
\hline LSCM1 & JN416253 & JN416202 & JN416154 & JN416304 & JN416356 \\
\hline LStr I & JN416256 & JN416205 & JN416157 & JN416307 & JN416359 \\
\hline LSCQ1 & JN416257 & JN416206 & JN416158 & JN416308 & JN416360 \\
\hline LSCR1 & JN416258 & JN416207 & JN416159 & JN416309 & JN416361 \\
\hline SCS1 & JN416259 & JN416208 & JN416160 & JN416310 & JN416362 \\
\hline
\end{tabular}

Table 4 GeneBank accession numbers for the sequenced isolates (Continued)

\begin{tabular}{lllccr}
\hline LSCT1 & JN416260 & JN416209 & $X$ & JN416311 & JN416363 \\
LSCU1 & JN416261 & JN416210 & JN416161 & JN416312 & JN416364 \\
\hline
\end{tabular}

there are substitutions in the amino acids on the viral capsid which may be relevant for the vaccination, iii) HPV infections with a variant may not give immunological protection against a subsequent infection with other variant of the same genotype.

Nowadays, HPV variants recombination has already been described and it is more often found since coinfection with more than one HPV type prevalence is not a unusual finding [10].

In our study we detected 2 recombinant variants (4.5\%) which might have been missed or wrong classified if only amplifying one genomic region. Furthermore, non-recombinant samples as LSCM or LSM3 showed specific-african substitutions in some regions (for example E6) whereas they would be classified as european variants when only analyzing nucleotide variation in URR. Therefore, characterizing more than one genomic region may be essential in order to detect recombination and classify HPV variants properly.

We amplified URR and E6, E7 and L1genes from at least $93 \%$ of samples. However, when characterizing E4 region, we were only able to amplify 35 samples. E4 gene is generally disrupted during DNA integration into the host genome and this disruption may explain the inability to amplify $E 4$ gene in some of our samples.

In conclusion, data and knowledge on geographic HPV intratypic variants distribution might help to establish a data base about the diversity and pathogenicity of different HPV variants, which may help to design and optimize diagnostics protocols in order to reduce the disease.

\section{Methods}

\section{Recruitment of participants}

Clinical Microbiology and Infection Control Department at Basurto University Hospital (Basque Country, North of Spain) analyzed samples which were remitted from different Hospital Services, especially the Consultation of Sexually Transmitted Diseases and the Department of Obstetrics and Gynecology, from 2007 to 2010.

All samples were collected from patients with clinical manifestations of HPV related infections. Lesions were classified by pathologists into three categories: negative (no lesion was found), low-grade squamous intraepithelial lesion (L-SIL) or high-grade squamous intraepithelial lesion (H-SIL).

Molecular genotyping was carried out using "Linear Array HPV Genotyping Test" kit (Roche Molecular 
Diagnostics). In our study, we analyzed positive samples for HPV genotype 18 (both single infections and multiple HPV infections) from patients who had given written, informed consent.

\section{Genomic DNA extraction}

DNA extraction was performed by QIAamp DNA mini Kit (Qiagen, Hilden, Germany), according to the manufacturer's instructions. Extracted DNA was eluted with $200 \mu \mathrm{l} \mathrm{AE}$ buffer and stored at $-20^{\circ} \mathrm{C}$ until amplification.

\section{PCR amplification and sequencing}

Amplification of HPV E6, E7, E4 genes and the URR was performed using type-specific primers designed according to HPV 18 genome prototype sequence (GenBank accession number NC001357). The URR was amplified using 2 primer sets. In order to amplify L1 region, consensus HPV primers were used (Table 3).

PCR was performed in $30 \mu \mathrm{l}$ of reaction mixture containing $10 \times$ PCR buffer, $25 \mathrm{mmol} / \mathrm{L} \mathrm{MgCl}_{2}, 25 \mathrm{mmol} / \mathrm{L}$ of each deoxynucleoside, $100 \mathrm{pmol} / \mathrm{L}$ of sense and antisense primer, $5 \mu \mathrm{l}$ of template DNA and 2,5 $\mathrm{U}$ of Taq DNA polymerase (Qiagen).

The thermal program started with a pre-heat of $95^{\circ} \mathrm{C}$ for 15 min, followed by 35-40 cycles of suitable annealing temperature which depended on the primers and finished with a final extension at $72^{\circ} \mathrm{C}$ for $10 \mathrm{~min}$ (Table 3).

PCR products were confirmed based on specific bands of amplified DNA presence in agarose gel (2\%). Afterwards, amplimers were automatically sequenced using the "Big Dye Terminator Cycle Sequencing kit" (Applied Biosystems) according to the manufacturer's instructions.

For E6, E7, E4 and URR amplicons the same forward specific primers as those used in amplification were chosen as sequencing primers. In the L1 region, a specific primer was used in order to sequence HPV 18 and not other HPV types present in cases of multiple infection (Table 3).

\section{Nucleotide variations, phylogenetic analysis: variants and recombination}

HPV sequences were aligned and compared to the HPV 18 prototype sequence which belongs to the Asianamerindian lineage (accession number NC001357), using BioEdit Sequence Alignment Editor v7.0.4.1 and Clustal W (http://www.genome.jp/tools/clustalw/).

Amplification and sequencing of the samples were repeated to confirm nucleotide variations which were present in less than three isolates.

Sequences were assigned to a lineage on the basis of their similarity to HPV 18 known variant sequences [26] which belong to Asian-amerindian lineage (GenBank accession numbers: EF202143 - EF202146), African (EF202152 EF202155) and European lineage (EF202147- EF202149,
EF202151). Phylogenetic trees were built using the Bayesian inference method implemented in MrBayes 3.1 [27] and three methods (all implemented in RDP3 [15]) were used for the detection of recombination (RDP [15], Maxchi [16] and Chimaera) to analyze isolates which did not adjust to the clusters.

\section{Lesions}

Association of lesions and variants or infection type (single HPV vs multiple HPV infection) was analyzed. Fisher exact test was used for statistically significant association.

\section{GenBank accession numbers}

The following are the GenBank accession numbers for all the sequences used in this analysis. $\mathrm{X}$ indicates absence of nucleotide sequence data Table 4.

\section{Ethical approval}

All procedures followed were approved by the appropriate Ethics Commitee related to our institutions (Basurto University Hospital and University of Basque Country) and complied with the guidelines and ethical standards for experimental investigation with human subjects of Helsinki Declaration of 1975, as revised in 2000. All study participants provided written, informed consent.

\section{Additional file}

Additional file 1: E4, E6, E7, L1, LCR_bay. Phylogenetic trees were constructed for each region individually.

\section{Abbreviations}

HPV: Human papillomavirus; H-SIL: High-grade squamous intraepithelial lesion; L-SIL: Low-grade squamous intraepithelial lesion; SIL: Squamous intraepithelial lesion; URR: Upstream regulatory region.

\section{Competing interests}

All authors declare no potential conflicts of interest.

\section{Authors' contributions}

LSA carried out the molecular studies, participated in the sequence alignment and drafted the manuscript. MB and EA participated in the design of the study and its coordination and have been involved in drafting the manuscript and revising it critically for important intellectual content. SH, DA and VE have made substantial contributions to acquisition of samples and data and have revised the manuscript critically. KGE and BMJ performed phylogenetic and statistical analysis. RC conceived the study, participated in its design and coordination and has given final approval of the version to be published. All authors read and approved the final manuscript.

\section{Acknowledgements}

Authors wish to thank the Department of Health from the Basque Government for supporting this project [project number 2008111058].

\section{Author details}

'Immunology, Microbiology and Parasitology Department, University of Basque Country, Leioa 48940, Spain. ${ }^{2}$ Clinical Microbiology and Infection Control Department, Basurto University Hospital, Bilbao 48013, Spain. ${ }^{3}$ Obstetrics and Gynecology Department, Basurto University Hospital, Bilbao 48013, Spain. ${ }^{4}$ Genetics, Physical Anthropology and Animal Physiology Department, University of Basque Country, Leioa 48940, Spain. 
Received: 4 June 2012 Accepted: 30 October 2012

Published: 2 November 2012

\section{References}

1. Muñoz N, Castellsague X, de Gonzalez AB, Gissmann L: HPV in the etiology of human cancer. Vaccine 2006, 24:1-10.

2. Smith JS, Lindsay L, Hoots B, Keys J, Franceschi S, Winer R, et al: Human papillomavirus type distribution in invasive cervical cancer and highgrade cervical lesions: a meta-analysis update. Int J Cancer 2007, 121:621-632.

3. Muñoz N, Bosch FX, Castellsagué X, Díaz M, de Sanjose S, Hammouda D, et al: Against which human papillomavirus types shall we vaccinate and screen? the international perspective. Int J Cancer 2004, 111:278-285.

4. Yamada T, Manos MM, Peto J, Greer CE, Munoz N, Bosch FX, et al: Human papillomavirus type 16 sequence variation in cervical cancers: a worldwide perspective. J Virol 1997, 71:2463-2472.

5. Ong CK, Chan SY, Campo MS, Campo MS, Fujinaga K, Mavromara-Nazos P, et al: Evolution of human papillomavirus type 18: an ancient phylogenetic root in Africa and intratype diversity reflect coevolution with human ethnic groups. J Virol 1993, 67:6424-6431.

6. de Araujo Souza PS, Sichero L, Macia PC: HPV variants and HLA polymorphisms: the role of variability on the risk of cervical cancer. Future Oncol 2009, 5:359-370.

7. Sichero L, Ferreira S, Trottier H, Duarte-Franco E, Ferenczy A, Franco EL, et al: High grade cervical lesions are caused preferentially by non-european variants of HPVs 16 and 18. Int J Cancer 2007, 120:1763-1768.

8. López-Savedra A, González-Maya L, de Ponce León S, García-Carranca A, Mohar A, Lizano M: Functional implication of sequence variation in the long control region and E2 gene among human papillomavirus type 18 variants. Arch Virol 2009, 154:747-754.

9. de Villiers EM, Fauquet C, Broker TR, Bernard HU, Zur HH: Classification of papillomaviruses. Virology 2004, 324:17-27.

10. Angulo M, Carvajal-Rodríguez A: Evidence of recombination within human alpha-papillomavirus. Virol J 2007, 4:33.

11. Jiang M, Xi LF, Edelstein ZR, Galloway DA, Olsem GJ, Lin WC, et al: Identification of recombinant human papillomavirus type 16 variants. Virology 2009, 394:8-11.

12. Méndez F, Muñoz N, Posso H, Molano M, Moreno V, van den Brule AJ, et al: Cervical coinfection with human papillomavirus (HPV) types and possible implications for the prevention of cervical cancer by HPV vaccines. J Infect Dis 2005, 192:1158-1165.

13. Tinelli A, Leo G, Dell'Edera D, Storelli F, Galante MM, Guido M, et al: Molecular methods for a correct diagnosis of multiple HPV infections and clinical implications for vaccine. Int J Gynecol Cancer 2011, 21:545-550.

14. Nielson CM, Harris RB, Flores R, Abrahamsen M, Papenfuss MR, Dunne EF, et al: Multiple-type human papillomavirus infection in male anogenital sites: prevalence and associated factors. Cancer Epidemiol Biomarkers Prev 2009, 18:1077-1083.

15. Martin DP, Lemey P, Lott M, Moulton V, Posada D, Lefeuvre P: RDP3: a flexible and fast computer program for analyzing recombination. Bioinformatics 2010, 26:2462-2463.

16. Smith J: Analyzing the mosaic structure of genes. J Mol Evol 1992, 34:126-129.

17. Xi LF, Kiviat NB, Hildesheim A, Galloway DA, Wheeler CM, Ho J, et al: Human papillomavirus type 16 and 18 variants: race-related distribution and persistence. J Natl Cancer Inst 2006, 98:1045-1052.

18. Im SS, Wilczynski SP, Burge RA, Monk BJ: Early stage cervical cancers containing human papillomavirus type 18 DNA have more nodal metastasis and deeper stromal invasion. Clin Cancer Res 2003, 9:4145-4150.

19. Burger R, Monk BJ, Kurosaki T, Anton-Culver H, Vasiley SA, Berman ML, et al: Human papillomavirus type 18: association with poor prognosis in early stage cancer. J Natl Cancer Inst 1996, 88:1361-1368.

20. Hecht J, Kadish A, Jiang G, Burk R: Genetic characterization of the human papillomavirus (HPV) 18 E2 gene in clinical specimens suggests the presence of a subtype with decreased oncogenic potential. Int J Cancer 1995, 60:369-376.

21. Villa LL, Sichero L, Rahal P, Caballero O, Ferenczy A, Rohan T, et al: Molecular variants of human papillomavirus types 16 and 18 preferentially associated with cervical neoplasma. J Gen Virol 2000, 81:2959-2968.

22. Rose B, Steger G, Dong XP, Thompson C, Cossart $Y$, Tattersall M, et al: Point mutations in SP1 motifs in the upstream regulatory region of human papillomavirus type 18 isolates from cervical cancers increase promoter activity. J Gen Virol 1998, 79:1659-1663.

23. Rose B, Thompson $\mathrm{CH}$, Zhang J, Stoeter M, Stephen A, Pfister $\mathrm{H}$, et al: Sequence variation in the upstream regulatory region of HPV 18 isolates from cervical cancers. Gynecol Oncol 1997, 66:282-289.

24. Meissner J: Sequencing errors in reference HPV clones. In Human papillomaviruses. A compilation and analysis of nucleic acid and amino acid sequences. 3rd edition. Edited by Myers G, Baker C, Munger K, et al. Los Alamos (NM): Theoretical Biology and Biophysics, Los Alamos National Laboratory; 1997:110-123.

25. Arias-Pulido H, Peyton CL, Torrez-Martinez N, Anderson DN, Wheeler CM: Human papillomavirus type 18 variant lineages in United States populations characterized by sequence analysis of LCR-E6, E2, and L1 regions. Virology 2005, 338:22-34.

26. Chen Z, DeSalle R, Schiffmn M, Herrero R, Burk RD: Evolutionary dynamics of variant genomes of human papillomavirus types 18, 45 and 97. J Virol 2009, 83:1443-1455.

27. Ronquist $F$, Huelsenbeck JP: MrBayes 3: Bayesian phylogenetic inference under mixed models. Bioinformatics 2003, 19:1572-1574.

doi:10.1186/1743-422X-9-258

Cite this article as: Arroyo et al:: Human Papillomavirus (HPV) genotype 18 variants in patients with clinical manifestations of HPV related infections in Bilbao, Spain. Virology Journal 2012 9:258.

\section{Submit your next manuscript to BioMed Central and take full advantage of:}

- Convenient online submission

- Thorough peer review

- No space constraints or color figure charges

- Immediate publication on acceptance

- Inclusion in PubMed, CAS, Scopus and Google Scholar

- Research which is freely available for redistribution 